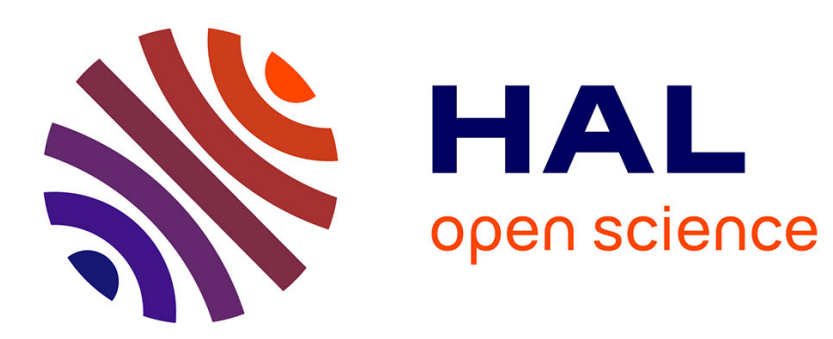

\title{
Joint Technology and Route Selection in Multi-RAT Wireless Sensor Networks with RODENT
}

\author{
Brandon Foubert, Nathalie Mitton
}

\section{To cite this version:}

Brandon Foubert, Nathalie Mitton. Joint Technology and Route Selection in Multi-RAT Wireless Sensor Networks with RODENT. IWCMC 2021 - 17th International Wireless Communications \& Mobile Computing Conference, Jun 2021, Harbin / Virtual, China. hal-03186044

\section{HAL Id: hal-03186044 https://hal.inria.fr/hal-03186044}

Submitted on 30 Mar 2021

HAL is a multi-disciplinary open access archive for the deposit and dissemination of scientific research documents, whether they are published or not. The documents may come from teaching and research institutions in France or abroad, or from public or private research centers.
L'archive ouverte pluridisciplinaire HAL, est destinée au dépôt et à la diffusion de documents scientifiques de niveau recherche, publiés ou non, émanant des établissements d'enseignement et de recherche français ou étrangers, des laboratoires publics ou privés. 


\title{
Joint Technology and Route Selection in Multi-RAT Wireless Sensor Networks with RODENT
}

\author{
Brandon Foubert and Nathalie Mitton \\ Inria - Lille, France \\ \{brandon.foubert, nathalie.mitton\}@inria.fr
}

\begin{abstract}
Wireless Sensor Networks (WSN) are limited by the characteristics of the Radio Access Technologies (RAT) their are based on. We call a wireless multi-hop network composed of nodes able to use several RAT a Multiple Technologies Network (MTN). Nodes must manage the RAT and route selection, in a local and distributed way, with an suitable communication protocol stack. Nodes may share multiple common RAT with multiple neighbors. Thus the devices' heterogeneity of technologies has to be taken into account by each of the stack's layer. In this article, we introduce our custom Routing Over Different Existing Network Technologies protocol (RODENT), designed for MTN. It is capable of dynamically (re)selecting the best RAT and route based on data requirements evolving over time. RODENT is based on a multi-criteria route selection via a custom lightweight TOPSIS method from our previous work [1]. For an evaluation of performance, we implemented a functional prototype of RODENT on Pycom FiPy devices. Results show that RODENT enables multiple data requirements support and energy savings, while increasing effective coverage.
\end{abstract}

Index Terms-LPWAN, WSN, MTN, RODENT, multi-RAT, heterogeneous, routing, multi-flow, Pycom FiPy

\section{INTRODUCTION}

Wireless Sensor Networks (WSN) allow many use cases such as remotely monitoring various metrics [2]. Such networks usually rely on a medium distance Radio Access Technology (RAT) (e.g., IEEE 802.15.4) and a multi-hop path routing. A specific subset of WSN, Low Power Wide Area Networks (LPWAN), usually rely on a long distance RAT (e.g., LoRaWAN) and star topology. This simplifies the network structure and enables wider coverage. When deployed, WSN usually use a single RAT shared by all nodes. The chosen RAT's limits, in terms of coverage and throughput, de facto constrain deployment. As an example, the Sigfox operatorbased network provide a very large range (up to $\mathrm{km}$ ) but does not offer a worldwide coverage. Specific data requirements such as large data or delay-intolerant data are impossible to withstand for the most constrained RAT. We also have to consider that outdoor nodes have to bear weather changes such as rain, which heavily alter the reliability of wireless links.

Actual WSN have trouble supporting multiple use-cases because of a lack of flexibility. Numerous RAT with various performances and capabilities are available nowadays [3]. Overcoming the aforementioned issues is possible with the use of Multiple Technologies Networks (MTN) [4]. Nodes could switch RAT and form multi-hop networks, which would extend the range of deployment. Based on the routes' performance and cost in energy, money etc., nodes would be able to select the RAT that best matches their needs. If the selected route's reliability or availability decreases, e.g., because of a change of weather or any node condition, nodes could select a new route with a different RAT. Nodes with several use-cases (e.g., video and humidity monitoring) could use different routes. The resiliency of the network would greatly increase, because in case of a RAT failure, nodes can use another technology.

Nodes should manage the RAT heterogeneity autonomously. But the routing protocols currently available does not support MTN. In this article, we introduce a novel Routing Over Different Existing Network Technologies protocol (RODENT) designed for MTN. It takes every RAT of a node into consideration for the routing process. As an input, it requires a list of the links available between the node and its neighbors. Such links have several associated performances and costs, in terms of energy consumption, delay, bit-rate etc. The routing table of each node is built based on the values from the list of its available links and the values from the routes shared by its neighbors. Nodes select the best route from the set of known routes by means of a custom TOPSIS method from our previous work [1]. The best route's criteria depend on the use case and the associated data requirements to fulfill (e.g., delay deadline, data size).

The performances of RODENT are assessed through implementation and experimental evaluation. To this end, we designed a MTN prototype based on Pycom FiPy devices embedding a custom MicroPython implementation of RODENT. Pycom FiPy nodes have multiple RAT available. Results show that with RODENT the network flexibility and reliability are increased. The energy consumption is lowered and data requirements are fulfilled at best, all while maintaining a good Packet Delivery Ratio (PDR). Unlike related work, RODENT provides a dynamic and flexible way to surpass the limitations of classic WSN without needing a dedicated infrastructure on the operator's side.

The rest of this paper is organized as follows: Section II presents the work related to MTN from the literature. Section III introduces the network model and assumptions we based RODENT on. Section IV exposes beforehand information about the methods used for route selection. Section V presents RODENT's inner workings. Section VI details the hardware and firmware used for our MTN prototype. Section VII presents the setup and scenario of the experiments. Section VIII details the results of the experiments. Section IX brings a conclusion to this article and lists future work. 


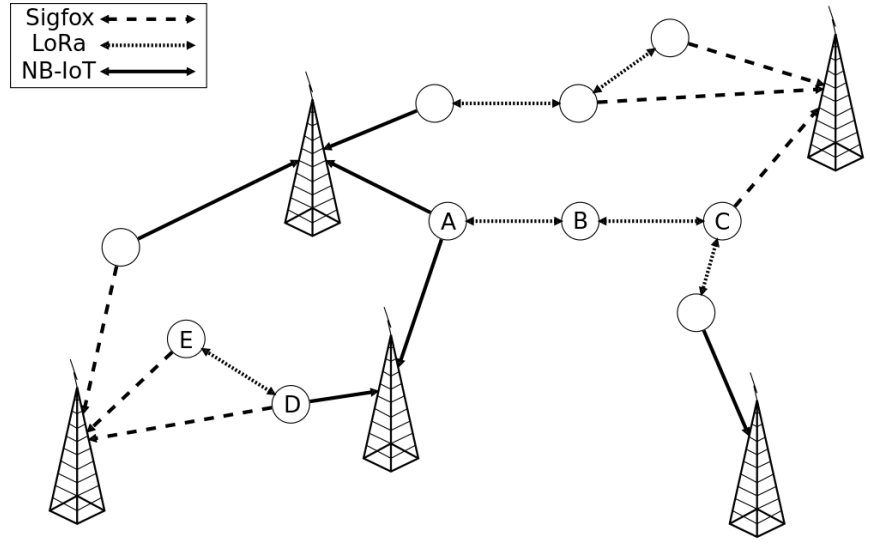

Fig. 1. MTN example.

\section{RELATED WORK}

To the best of our knowledge, there is few work in the literature about multi-RAT networks. In this section we present the work related to multi-technologies devices.

In [5] the authors propose a multi-RAT IoT architecture for devices. It is based on a network convergence layer managing the multi-RAT aspect from the nodes, and a heterogeneous network controller on the network operator side. An hardware platform for the nodes is also proposed, with specific polling and compression schemes based on Static Context Header Compression (SCHC). This efficiently increases the network flexibility, however it requires a specific virtual network operator. The authors of the next work [6] propose a virtual network operator based on the cloud for multi-modal LPWA networks. The virtual network operator manage the configuration and operations of the heterogeneous LPWAN hardware. As in the previous work, it requires the deployment of a specific infrastructure on the operator side.

The authors of [7] introduce a green path inter-MAC selection protocol. The protocol enables MAC layer path selection, to minimize energy consumption and radio frequency utilization. However, no information about the routing layer is given as this is out of the article's scope. In [8] the authors detail the ORCHESTRA framework. It manages real-time intertechnology handovers. The framework is based on a virtual MAC layer, coordinating every layers from each technology with a single MAC address. As for the previous work, this one also focuses on the link layer and omits the routing layer.

WSN's flexibility is increased in every one of the aforementioned works. But as they require a dedicated infrastructure, it brings in turn a new limitation. In this article, we present a routing protocol adapted to MTN, which greatly increase WSN's capabilities while requesting only multi-RAT nodes.

\section{MODEL \& ASSUMPTIONS}

RODENT's design is based on a specific network model and several assumptions about the communication stack's lower layers. In this section we describe those model and assumptions.
TABLE I

EXAMPLE LINK MATRIX $L M_{D}$.

\begin{tabular}{|l|c|c|c|}
\hline & Energy & Money & Bit-rate \\
\hline Sigfox BS & 12 & 102 & 22 \\
\hline NB-IoT BS & 151 & 87 & 174 \\
\hline Node E (LoRa) & 37 & 0 & 72 \\
\hline
\end{tabular}

\section{A. Network model}

The nodes taking part in a WSN usually follow one or multiple traffic patterns [9]. Here, we assume that the nodes running RODENT communicate only in a convergecast pattern, from the nodes to the sink. As the nodes of a MTN have multiple RAT, there can be several links between a single pair of nodes. We assume that the network forms a connected graph if we consider every link independently of their RAT. Several data requirements can be fulfilled by the same node (e.g., monitoring and alarm) as long as those requirements are known by every node in the MTN. An example of a MTN is shown in Figure 1. Here, node B $\left(N_{B}\right)$ monitors temperature but is not in the vicinity of a Sigfox or NB-IoT base station. Since $N_{B}$ has multiple RAT, it can forward its data to $N_{A}$ or $N_{C}$ through a LoRa link. They can then offload $N_{B}$ 's data to a base station using another RAT.

\section{B. Data requirements}

We want RODENT's nodes to support multiple use cases, i.e., multiple purposes (e.g., monitoring the weather, record videos). Needs and data requirements are different for each use case. To send a video, a RAT with a high bit-rate is essential to ensure low delay and jitter. To send an alarm, a RAT with a short delay is needed, but the bandwidth is secondary. To regularly send a small amount of numerical monitoring data, a RAT with a low power consumption is the priority. Several data requirements can be supported on a single node e.g., regularly sending the amount of rainfall and an alarm in case of a flood. The aim of the route selection is to satisfy at best the nodes' data requirements.

\section{Assumptions on communication stack}

In this article, we focus on the network layer, specifically routing. We assume that the communication stack's lower layers run protocols suited to MTN. We assume that the physical and link layers can assess the availability and reliability of the links between a node and its neighbors (i.e., nodes or base stations) for every RAT. We consider that those layers gather or estimate information about the performances and costs of each link (i.e., energy consumption, bit-rate, etc.), as WSN radio link quality estimation is a well studied subject [10].

As input, RODENT takes a table or matrix of links, to which we refer to as $L M_{i}$ for node $i$. The size of $L M_{i}$ depends on the number of characteristics considered, the number of available RAT and the number of $i$ 's neighbors. As an example, in Figure $1 N_{D}$ could have a $L M_{D}$ such as Table I. $L M_{D}$ is comprised of every link between $N_{D}$ and its neighbors as well as the links' characteristics. 
TABLE II

EXAMPLE ROUTE MATRIX $R M_{D}$.

\begin{tabular}{|l|c|c|c|c|}
\hline & Energy & Money & Bit-rate & Hops \\
\hline Sigfox BS & 12 & 102 & 22 & 1 \\
\hline NB-IoT BS & 151 & 87 & 174 & 1 \\
\hline Node E $($ LoRa $)$ & 49 & 102 & 94 & 2 \\
\hline
\end{tabular}

\section{BACKGROUND: SELECTION METHOD}

In MTN, a single node owns several RAT. This hardens the route selection as nodes must consider many routes over many RAT. In this section, we present RODENT's route selection method based on our previous work [1].

The selection process has to take account of multiple criteria e.g., the energy consumption, delay etc. of each route to meet as much as possible all data specific requirements. Several tools are available in the literature for multi-criteria decision, such as utility and cost functions, Markov chains, fuzzy logic, game theory, data mining and Dempster-Shafer theory. We found the Multiple Attribute Decision Making (MADM) methods to be the most fitting for route selection.

With MADM methods, the problem is formalized as a decision matrix. It is composed of the candidates and their attributes. A set of attributes' values is associated to each candidate, reflecting its performance. The decision matrix serves as input of an MADM method, which outputs the candidates' ranking. Several MADM methods are available in the literature, such as Simple Additive Weighting (SAW), Weighting Product (WP), Analytical Hierarchy Process (AHP) and Gray Relational Analysis (GRA). Among those methods, we find the most interesting to be Technique for Order Preference by Similarity to Ideal Solution (TOPSIS). TOPSIS allows the comparison of each candidate based not only on its closeness to the best theoretical candidate but also on its distance from the worst theoretical candidate.

Classic TOPSIS has two main issues: rank reversal and complex ranking computation algorithm. We use a lightweight TOPSIS for WSN from our previous work [1] which is designed for hardware-constrained devices rand removes rank reversals. We leverage this lightweight TOPSIS method to operate the route selection in RODENT.

For node $i$, its route matrix is referred to as $R M_{i}$. Considering route selection, all the routes available for node $i$ are stored in $R M_{i}$. The attributes in $R M_{i}$ are relative to the routes e.g., the expected transmission count, the total energy consumption of the number of hops. As an example, in Figure 1 node $D$ could have $R M_{D}$ such as Table II. A set of weights relative to each attribute is required as input by TOPSIS. Those represent the importance of each attribute in the ranking process. We call a set of weights a Requirements Vector $(R V)$. For use case $x, R V_{x}$ is its requirements vector (e.g., $\left.R V_{\text {monitoring }}\right)$. Considering route selection, the values of a $R V$ are based on the data requirements nodes have to fulfill (e.g., prioritize speed over energy consumption), and such that $R V\left\{e_{n} \in R V \mid \sum_{n=1}^{|R V|} e_{n}=1\right\}$. An example of requirements vector is shown in Table III.
TABLE III

REQUIREMENTS VECTORS.

\begin{tabular}{|c|c|c|c|}
\hline & Energy & Money & Bit-rate \\
\hline$R V_{\text {monitoring }}$ & 0.6 & 0.3 & 0.1 \\
\hline$R V_{\text {alarm }}$ & 0.1 & 0.1 & 0.8 \\
\hline
\end{tabular}

\section{ROUTING OPERATIONS}

RODENT's distinctive feature is to allow the use of multiRAT routes. Each route has different performance and costs. In this section, we detail the routing operations performed by RODENT. We introduce the following notations that are used further in the paper. We refer to node $i$ as $N_{i}$. Nodes in the direct vicinity of $N_{i}$ are called neighbors, and we refer to the set of neighbors as $N B R(i)$ for $N_{i}$. NBR $(i)_{j}$ is $N_{j}$ such that $N_{j} \in N B R(i) . N B R(i)_{j}$ has at least one link with $N_{i}$, which we refer to $L_{i j}^{x}$ for RAT $x$. The route of $N_{i}$ that goes through $L_{i j}^{x}$ is referred to as $R_{i j}^{x}$.

\section{A. Overview}

As an example we can consider the operations of $N_{D}$ and $N_{E}$ from Figure 1. $N_{D}$ boots without information about its surroundings. The link layer of $N_{D}$ probes the links with every RAT and builds $L M_{D}$ similar to Table I. The network layer then builds the route matrix $R M_{D}$ based on $L M_{D}$. Every direct links from $N_{D}$ to a base station are inserted in $R M_{D}$ as singlehop routes. $N_{E}$ does the same and selects the only route it has toward the Sigfox base-station. This route is advertised and received by $N_{D}$ through $L_{E D}^{L o R a} . N_{D}$ 's third route is built based on the link's and route's values. $R M_{D}$ is then similar to Table II. Then for each of its $R V, N_{D}$ selects a best route, based on the routes' values, independently of the RAT. Here, if we consider Table III, for $R V_{\text {monitoring }}$ low energy consumption is favored, thus the route toward the Sigfox base station is selected. For $R V_{\text {alarm }}$ a high bit-rate is favored, thus the route toward the NB-IoT base station is chosen. Finally, $N_{D}$ starts to use and advertise its best routes.

\section{B. Packet structure}

The structure of a RODENT's packet is shown in Figure 2. A packet is composed of three parts: $(i)$ the header (ii) the payload (iii) the trailer. RODENT's control data is found in the header. The Network Identifier is a two byte value known by every node in the MTN which is used to recognize RODENT's packets. The Source Identifier is a two byte value equal to the unique ID of the packet's source node. The Destination Identifier is a two byte value equal to the unique ID of the packet's destination node. The Payload Size is a one byte value equal the size in bytes of the payload. The Requirement Vector Identifier is a one byte value indicating the type i.e., use case of the data contained in the payload. The Route is a four byte array with the best route's values i.e., energy, money, bit-rate and number of hops from $N_{\text {Source Identifier. The payload is a series of Payload Size }}$ bytes which corresponds to the data shared by the source. Fi- 
nally, the trailer is a single byte equal to the CRC8 Checksum of the header and payload parts.

\section{Route construction}

Here we consider the routing operations of $N_{i} . N_{i}$ boots up and begins the construction of $R M_{i}$. RODENT uses two data sets: $L M_{i}$ the link matrix of $N_{i}$ and the routes shared by $N B R(i)$. The first step of $N_{i}$ is to search $L M_{i}$ for any link from $N_{i}$ to a base station e.g., a Sigfox antenna of LoRaWAN gateway. Such links are directly converted to single hop routes based on the values found in $L M_{i}$ and stored in $R M_{i}$. The second step of $N_{i}$ is to build the routes passing through the nodes of $N B R(i)$. Considering a route coming from $N B R(i)_{j}, N_{i}$ adds the attributes' values of its link $L_{i j}^{x}$ to the attributes' values of the received route. The new route $R_{i j}^{x}$ is then appended to $R M_{i}$.

\section{Route selection}

The route selection in classic WSN is usually trivial, as the route of lowest cost or rank is chosen. In an MTN, a route is a succession of links where each link potentially use a different RAT. Various performances are offered by different RAT. As we aim to support multiple use cases with different data requirements, the route selection must take into account multiple criteria. Section IV introduces the selection method of RODENT. Considering $N_{i}$, our lightweight TOPSIS method takes as input $R M_{i}$ and a requirement vector $R V_{x}$ relative to use case $x$. A ranking of the routes comes out of the selection method, and the route on top is the one fulfilling at best the data requirements of use case $x$. For $N_{i}$ and for a use case $x$ we refer to the best route as $B R_{i}^{x}$.

\section{E. Route propagation}

The propagation of the routes is made with two mechanisms: piggybacking and control packets. To share routes without the need of dedicated transmissions we use piggybacking. In a RODENT packet from $N_{i}$ and relative to use case $x$, the header carries the ID number of $R V_{x}$ and the best route $B R_{i}^{x}$. Because wireless communications share a common medium, $N_{i}$ overhears the packets from $N B R(i)$. This allows $N_{i}$ to opportunistically update $R M_{i}$. If $N_{i}$ does not overhears $R_{i j}^{x}$ from $N B R(i)_{j}$ anymore (e.g., because of $N_{j}$ 's failure), $R_{i j}^{x}$ will timeout and will be deleted from $R M_{i}$. To keep alive the unused routes, $N B R(i)_{j}$ can use dedicated control packets. Control packets are regular packets with an empty payload.

\section{IMPLEMENTATION}

RODENT's implementation is done on FiPy devices from Pycom [11]. Those devices' specificity is the availability of five different RAT. These nodes form an MTN and offload their data to WiFi and LoRa base stations (BS). The experiments' hardware and firmware are presented in this section.

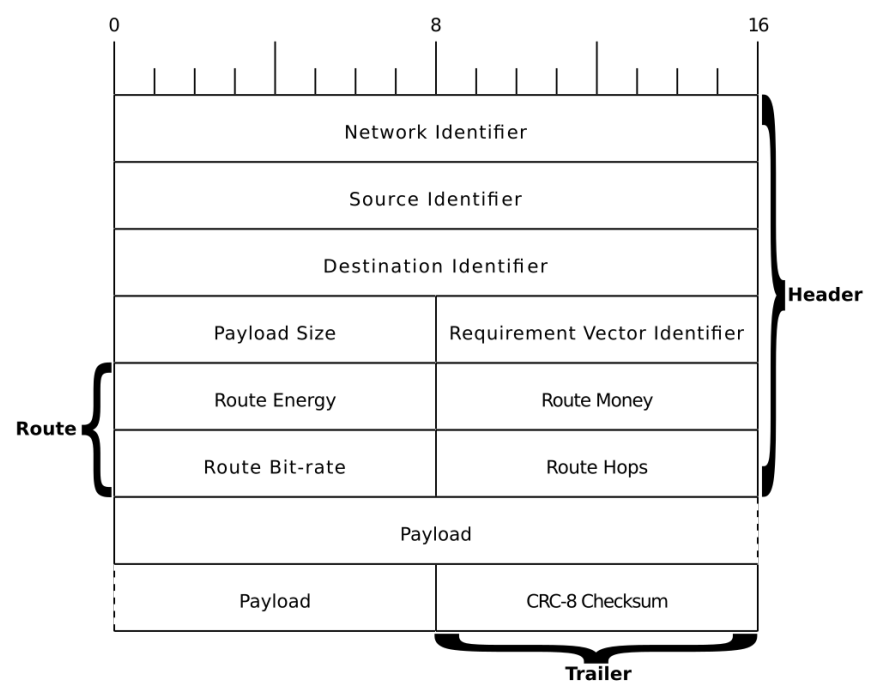

Fig. 2. RODENT packet structure.

\section{A. Hardware}

The FiPy nodes from Pycom are made from WSN hardware: CPU ESP32, several wireless RAT, few memory available and low power consumption. The different RAT embedded in a FiPy are LTE-M, NB-IoT, Sigfox, LoRa, Bluetooth Low Energy (BLE) and WiFi. Each one offers a different performance in terms of bit-rate, energy consumption, economical cost, etc., which are considered by the route selection of RODENT. Here we coupled the FiPy nodes with Pytrack sensor shields which offer a GPS, an accelerometer and a micro-USB port.

A B-L072Z-LRWAN1 board [12] is used as a LoRa BS. An Edimax EW-7811Un dongle [13] serves as a WiFi BS. Every device is connected to a Trip Lite U223-007 (7-Port USB Hub). The main computer, a Dell Latitude 5590, powers devices, collects and analyses results.

\section{B. Firmware}

Based on the FiPy's firmware (a MicroPython port), we implemented RODENT in Python. After the boot sequence, $N_{i}$ computes its unique ID. The needed RAT are booted based on $L M_{i}$ and the building of routes starts. The node's main loop is: $i$ ) select the best route for each $R V_{x}$, ii) add the next payload to the transmission buffer iii) send every payload from the buffer. The routes received from $N B R(i)$ are appended to $R M_{i}$. The payloads received from $N B R(i)$ are inserted into the transmission buffer. The keep track of operations, nodes print the sent packets' data on the serial port. The switch between $R V$ happen upon a press of the Pytrack's button. The implemented $R V$ are $R V_{\text {monitoring }}$ and $R V$ alarm.

The firmware of the LoRa BS is implemented in C. It listens constantly for LoRa transmissions. LoRa transmissions of RODENT's packets are unpacked and printed on the serial port. The firmware of the WiFi BS is coded in Python. WiFi transmissions of RODENT's packets are unpacked and printed on stdout. 


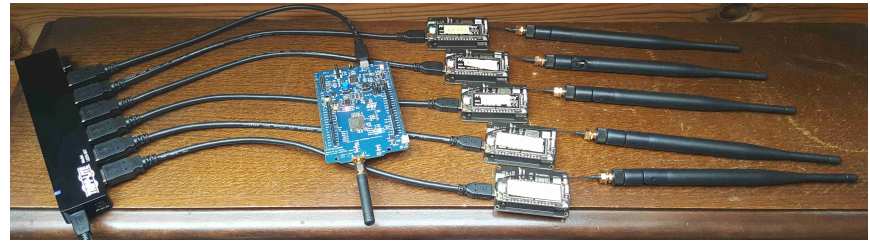

Fig. 3. Experimental setup.

\section{EXPERIMENTS}

We use real hardware to run experiments to assess the performance of RODENT. The nodes are configured to unroll a specific scenario and monitor the results. The scenario and setup of the experiments are detailed in this section.

\section{A. Setup}

The devices described in Section VI are connected to the USB hub which in turn is connected to the main computer. Nodes and BS are powered at the same time and boot up immediately. Every device is sitting very close the each other as shown in Figure 3. The stdout of the WiFi BS and the serial ports of the nodes and the LoRa BS are monitored by the main computer. The results' computation is done post-experiment.

\section{B. Scenario}

The use case we simulate is the monitoring of a farm. In this scenario, we monitor a field used for cultivating crops with nodes. The simulated setup is shown in Figure 4. The useful environmental metrics are measured by five nodes. These nodes have to regularly offload numerical data while saving up power. If a metric becomes off chart (e.g., temperature) and puts the crops at risk, they have to send an alarm.

Five RAT are available on FiPy nodes, but we only use WiFi, LoRa and BLE. Sigfox and LTE-M/NB-IoT aren't open technologies, thus it would considerably harden the experiments. WiFi links require more energy than LoRa and BLE links. Each node $N_{i}$ shows a different situation. $N_{1}$ is the control node and can only use its WiFi link toward the WiFi BS. $N_{2}$ have to chose between its WiFi and LoRa links to the base stations. $N_{3}$ can either reach the WiFi BS or use its BLE link with $N_{1}$ to forward data at a lower energy cost. $N_{4}$ have to send monitoring data but also alarms, and can do so through its WiFi and LoRa links with the base stations. $N_{5}$ is an isolated node, too far from the WiFi BS to have a direct link. Since farms are usually wide rural environments where tall crops like corn are unfriendly to wireless wave, white zones and isolated nodes are common. But thanks to RODENT, $N_{5}$ has the ability to use LoRa to reach $N_{4}$.

Three types of experiments were ran: (i) RODENT is inactive and only the WiFi links are available, which are shown in blue on Figure 4. (ii) RODENT is active and the LoRa and BLE links are available, shown in red and green in Figure 4. (iii) RODENT is active, LoRa messages are sent two times, BLE messages three times, to increase the network's reliability. A video of an experiment is available online ${ }^{1}$.

\footnotetext{
${ }^{1}$ http://chercheurs.lille.inria.fr/bfoubert/ressources/rodent.mp4
}

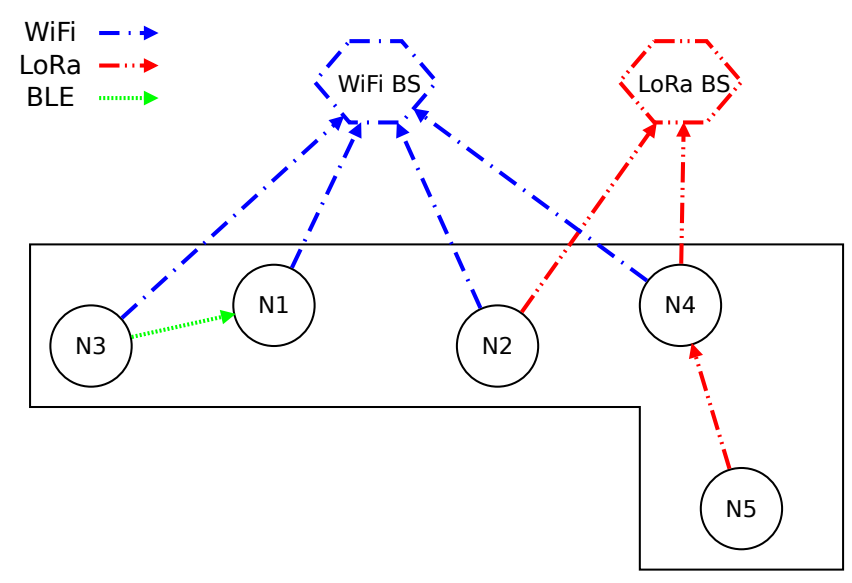

Fig. 4. Farm monitoring scenario.

\section{RESULTS}

We measure the topology and Packet Delivery Ratio (PDR). The transmission interval of the nodes is picked randomly in $[2 ; 4]$ seconds. We consider the results of 20 experiments of a duration of 10 minutes each. A small number of experiments is sufficient because the standard deviation is low. A longer duration is not relevant as after only a few messages, the network stabilizes. Since related works' proposals differ a lot from ours, and an increase in flexibility cannot be measured, we do not directly compare their results with RODENT. In this section, we present the experiments' results we've obtained.

\section{A. Topology}

The topology of the MTN changes when RODENT is enabled. $N_{1}$ can only reach the WiFi BS, thus it keeps the same link. For monitoring, the nodes want to save up as much energy as possible. Thus, $N_{2}$ switches from the WiFi link to the LoRa link. $N_{3}$ starts to offload its data to $N_{1}$ thanks to their BLE link, and $N_{1}$ forwards the data to the WiFi BS. $N_{4}$ chooses to offload its monitoring data to the LoRa BS, but can still use its route toward the WiFi BS to send or forward alarms, which need a quick RAT even if it costs more energy. $N_{5}$ comes out from its isolation and can now forward data through $N_{4}$ using LoRa. In turn, $N_{4}$ forwards $N_{5}$ 's data to the LoRa BS or WiFi BS depending on the data requirements.

\section{B. Packet Delivery Ratio}

The ratio between the total packets received and the total packets sent is known as the Packet Delivery Ratio (PDR). The Figure 5 shows the PDR and its standard deviation for every node. We see that the PDR of $N_{1}$ is stable, as it keeps the same route. When RODENT is inactive, the PDR of $N_{5}$ is null because it can't offload a single data packet. With RODENT active, the PDR of $N_{2}, N_{4}$ and $N_{5}$ is around $80 \%$ because they use LoRa. Here nodes use LoRaRAW, without a proper MAC, which make collisions more frequent. With RODENT, the PDR of $N_{3}$ is around $60 \%$, because it forwards its data through BLE to $N_{1}$. BLE isn't made for single message 


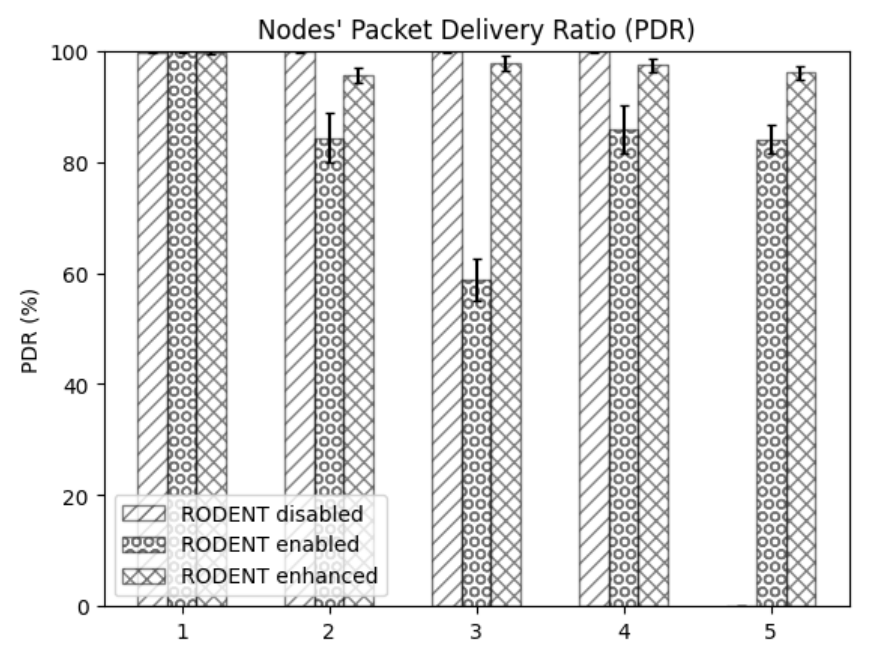

Fig. 5. Packet Delivery Ratio per node.

transmission, thus we tweaked single BLE advertisements to achieve it, hence the packet losses. When enhancing RODENT, every node achieve a better PDR, very close to the PDR obtained with only WiFi.

\section{Energy consumption}

The FiPy nodes from Pycom suffer from some design problems. This makes physical measurement of energy consumption hazardous [14]. To get a general idea, we choose to stick to the energy ratings from the components datasheets [11], [15]. These are showed in Figure 6. BLE needs approximately half-less current than $\mathrm{WiFi}$, and LoRa a tenth. WiFi and BLE offer the same bit-rate with the Pycom's CPU. The bit-rate of LoRa is much slower, which means longer transmission for the same amount of data. However, WiFi and BLE require a lot more traffic control than LoRa, which allows LoRa to use less energy. We can thus assume that RODENT enables significant energy savings.

\section{CONCLUSION}

The coverage and other performance of RAT limit WSN deployment. Conceiving multi-RAT devices and deploying MTN can overcome these limitations. In this article, we introduce the novel Routing Over Different Existing Network Technologies protocol (RODENT). It allows multi-technologies routing in MTN. With a RODENT embedded prototype we demonstrate the utility and feasibility of MTN. From the experiments' results we see that RODENT increase the network's reliability, flexibility and energy savings while maintaining a good PDR. This work however lacks a study about the scalability.

Thus, as future work, we plan to run large experiments in real fields to test the scalability and practicality of RODENT. We want to extend RODENT to support downlink communication and precisely measure the energy consumption of nodes. This aim to further increase the nodes' flexibility and use cases (e.g., firmware over the air upgrade). We also

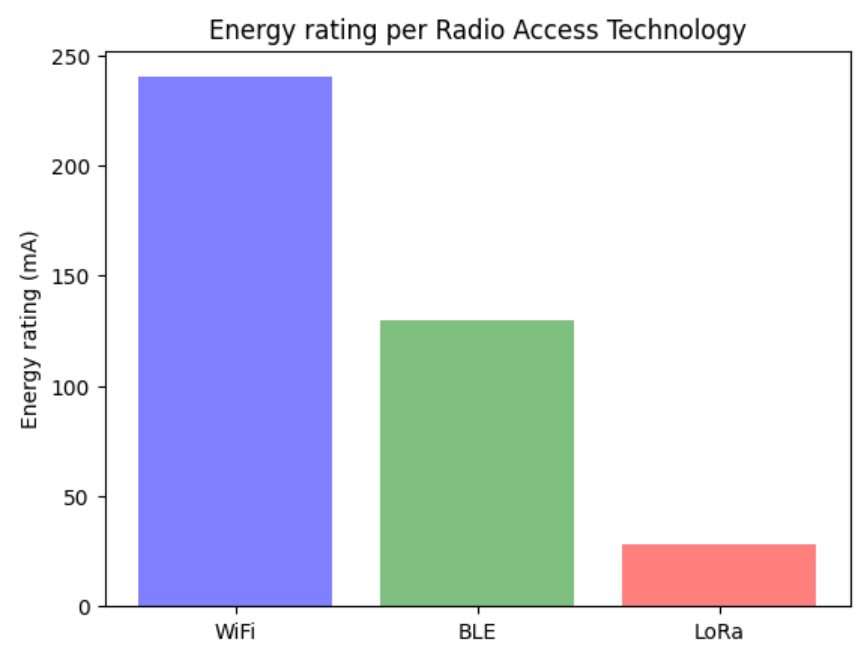

Fig. 6. Energy consumption per RAT.

wish to design an efficient link layer protocol for precise link reliability assessment adapted to MTN.

\section{ACKNOWLEDGMENTS}

This work was partially supported by a grant from CPER DATA, Sencrop, FEDER, I-SITE and Lirima Agrinet project.

\section{REFERENCES}

[1] B. Foubert and N. Mitton, "Lightweight network interface selection for reliable communications in multi-technologies wireless sensor networks," in $D R C N, 2021$.

[2] D. Ye, D. Gong, and W. Wang, "Application of wireless sensor networks in environmental monitoring," in PEITS, 2009.

[3] B. Foubert and N. Mitton, "Long-range wireless radio technologies: A survey," Future Internet, vol. 12, no. 1, 2020.

[4] B. Foubert and N. Mitton, "Autonomous Collaborative Wireless Weather Stations: A Helping Hand for Farmers," ERCIM News, no. 119, pp. 37 38, 2019.

[5] J. Famaey, R. Berkvens, G. Ergeerts, E. D. Poorter, F. V. d. Abeele, T. Bolckmans, J. Hoebeke, and M. Weyn, "Flexible multimodal subgigahertz communication for heterogeneous internet of things applications," IEEE Communications Magazine, vol. 56, no. 7, pp. 146-153, 2018.

[6] J. Hoebeke, J. Haxhibeqiri, B. Moons, M. Van Eeghem, J. Rossey, A. Karagaac, and J. Famaey, "A cloud-based virtual network operator for managing multimodal lpwa networks and devices," in CIoT, 2018.

[7] O. Bouchet, A. Kortebi, and M. Boucher, "Inter-mac green path selection for heterogeneous networks," in Globecom Workshops, 2012.

[8] T. De Schepper, P. Bosch, E. Zeljković, F. Mahfoudhi, J. Haxhibeqiri, J. Hoebeke, J. Famaey, and S. Latré, "Orchestra: Enabling intertechnology network management in heterogeneous wireless networks," IEEE Transactions on Network and Service Management, vol. 15, no. 4 , pp. 1733-1746, 2018.

[9] N. A. Pantazis, S. A. Nikolidakis, and D. D. Vergados, "Energyefficient routing protocols in wireless sensor networks: A survey," IEEE Communications Surveys Tutorials, vol. 15, no. 2, 2013.

[10] N. Baccour, A. Koubâa, L. Mottola, M. A. Zúñiga, H. Youssef, C. A. Boano, and M. Alves, "Radio link quality estimation in wireless sensor networks: A survey," ACM Trans. Sen. Netw., vol. 8, no. 4, 2012.

[11] FiPy Specsheet, Pycom, 2018, version 1.0.

[12] UM2115 User manual, STMicroelectronics, 2018, rev 5.

[13] EW-7811Un Datasheet, Edimax, 2015.

[14] T. Andersén, "Energy-efficient adaptive sensing in low power wide area networks," Master's thesis, NTNU, 2018.

[15] ESP32 Series Datasheet, Espressif Systems, 2020, version 3.3. 